\title{
A Magnetic Field-to-Digital Converter Employing a Spin-Torque Nano-Oscillator
}

\author{
Dagur I. Albertsson, Johan Åkerman, and Ana Rusu
}

\begin{abstract}
In this work, a novel magnetic field-to-digital converter based on emerging spin-torque nano-oscillators (STNOs) is proposed. The architecture is inspired by voltage controlled oscillator (VCO)-based analog-to-digital converters (ADCs) which have shown inherent first-order noise shaping of both quantization- and phase-noise without the need for feedback. In the proposed architecture, the STNO acts both as a magnetic field sensor and VCO. The architecture's performance is evaluated in terms of signal-to-noise and distortion ratio (SNDR) utilizing Verilog-AMS modeling, where a macrospin model fitted to experimental data is employed for accurate description of the STNO operation. The presented simulation results demonstrate the potential of the STNO-based magnetic field-to-digital converter architecture.
\end{abstract}

Index Terms-Spin-torque Nano-Oscillator, Spintronics, Verilog, Magnetic field sensor.

\section{INTRODUCTION}

$\mathbf{O}$ VER the last decades, sensor based systems have revolutionized many industries. Specifically, magnetic field sensors are used in magnetic storage devices [1], biosensing applications [2], structural health monitoring [3], automobiles and a wide range of applications in factories [4]. The most common magnetic field sensors employed in these applications include Hall-effect, anisotropic-, giant- and tunnelingmagnetoresistance sensors [5]. Alternatively, a relatively new type of magnetic field sensors that might allow for higher spatial resolution are based on spin-torque nano-oscillators (STNOs) [6], a novel oscillator which has attracted significant attention in recent years. Their nano-scale size along with wide frequency tunability make STNOs an interesting device for a number of applications, e.g. wireless communication systems [7], neuromorphic/non-boolean computing [8], [9] and as magnetic storage read/write probes [10], [11] or magnetic field sensors [6]. However, the STNOs current performance in terms of output power and phase noise limits their applications. The STNO applications often rely on monitoring the operating frequency which brings challenges for the analog blocks in the readout where wide bandwidth, low noise and high gain are needed. Specifically, STNO magnetic field sensors are based on tracking changes in the operating frequency, which can be multi-GHz for small variations in magnetic

This work was supported by the Swedish Research Council VR.

D. I. Albertsson and A. Rusu are with the Department of Electrical Engineering, School of EECS, KTH Royal Institute of Technology, Kista, Sweden.

J. Akerman is with the Department of Physics, University of Gothenburg, Gothenburg, Sweden.

Copyright (c) 2020 IEEE. Personal use of this material is permitted However, permission to use this material for any other other purposes must be obtained from the IEEE by sending a request to pubs-permissions@ieee.org .

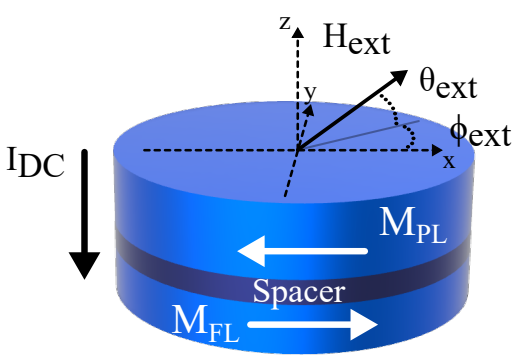

(a)

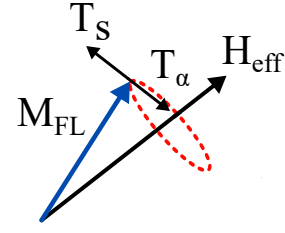

(b)
Fig. 1. (a) Illustration of the MTJ STNO and (b) Graphical representation of terms in the LLGS equation.

fields. Consequently, STNOs have the potential of being used as high resolution magnetic field sensors. A typical readout circuitry for STNO-based magnetic field sensors proposed in [12] requires a frequency-to-voltage converter followed by an analog-to-digital converter (ADC), which are challenging to design and consume substantial area and power. In this work, we take a step further by proposing a potentially more power and area efficient readout architecture, which is a STNO-based magnetic field-to-digital converter. The proposed architecture is inspired by voltage controlled oscillator (VCO)based ADCs, which are mostly digital CMOS circuits that bring the advantages of low power, small area and technology scaling [13], [14].

The paper is organized as follows. Section II describes the fundamental characteristics of STNO devices and the proposed architecture. Section III covers simulation results and discusses possible performance enhancements of the magnetic field-todigital converter. Finally, section IV draws conclusions.

\section{Proposed Magnetic Field-to-Digital CONVERTER}

\section{A. STNO operation}

Spintronic oscillators are nanoscaled, current controlled high frequency devices which can be categorized in terms of material structure and/or geometry [15]. The most common STNOs are either based on magnetic tunnel junctions (MTJs) or spin-valves. MTJs are made of an insulator spacer, such as $\mathrm{MgO}$, sandwiched between two ferromagnetic layers (typically $\mathrm{CoFeB}$ or NiFe), as it is shown in Fig. 1.(a), while in spinvalves the insulator is replaced by a non-magnetic metal spacer such as $\mathrm{Cu}$. These devices feature different characteristics where MTJ based STNOs have shown higher output power while spin-valve oscillators have a wider frequency range [15]. 
Due to the higher output power of MTJ STNOs, they have gained more interest in recent years [15]. The MTJ STNO is composed of a pined ferromagnetic layer (PL) designed to have a constant magnetization direction while the free layer (FL) can precess when a magnetic field, $H_{e x t}$ and a DC current, $I_{D C}$ are applied to the device. The precession is explained in terms of the Landau-Lifshitz-Gilbert equation with a Slonczewski spin-transfer (LLGS) term as [16], [17]:

$$
\frac{d \mathbf{M}_{\mathbf{F L}}}{d t}=-\gamma\left[\mathbf{M}_{\mathbf{F L}} \times \mathbf{H}_{\mathrm{eff}}\right]+\mathbf{T}_{\alpha}+\mathbf{T}_{\mathbf{S}}
$$

where $\mathbf{M}_{\mathbf{F L}}$ is the FL magnetization direction, $\gamma$ is the gyromagnetic ratio, $\mathbf{H}_{\mathbf{e f f}}$ is the effective magnetic field inside the FL, $\mathbf{T}_{\alpha}$ is the magnetization intrinsic damping torque and $\mathbf{T}_{\mathbf{S}}$ is an antidamping torque as shown in Fig. 1.(b). When a DC current, $I_{D C}$, is passed through the STNO, the electrons become spin-polarized in the direction of magnetization inside the PL, tunnel through the insulating spacer and exert a torque $\mathbf{T}_{\mathbf{S}}$ on the FL. This torque is called spin-transfer torque (STT) and is proportional to both the angle between local magnetization within each layer and the STT polarization [16], [18]. Consequently, a spin-polarized DC current can be used to cancel out the magnetization damping $\mathbf{T}_{\alpha}$ through $\mathbf{T}_{\mathbf{S}}$ and excite steady state magnetization precission as it is described in Eq. (1) and Fig.1.(b).

The tunneling magnetoresistance (TMR) effect [19], [20] translates the STT induced magnetization precession into an output voltage with a frequency proportional to the field and DC current, described as [21]:

$$
V_{S T N O}=\left(R_{d c}+R_{\text {prec }} \cos \left(2 \pi f_{S T N O} t+\phi\right)\right) I_{D C}
$$

where $R_{d c}$ is the MTJ DC resistance, $R_{\text {prec }}$ is peak precessional resistance induced by the FL magnetization oscillations, $f_{S T N O}$ is the operating frequency and $\phi$ the phase noise. Both $R_{\text {prec }}$ and $f_{S T N O}$ are strongly affected by changes in both the DC current and externally applied magnetic field $H_{\text {ext }}$ (direction and magnitude). Therefore, the external magnetic field can be measured by monitoring the changes in the STNO operating frequency, which have shown a linear relationship [22]. These characteristics offer the possibility of utilizing STNOs as frequency based nanoscaled magnetic field sensors.

\section{B. $V C O A D C$}

Time-based circuits, which process analog signals in time domain rather than voltage domain, have gained substantial interest in recent years. The main driving force has been technology scaling, where signal processing in the voltage domain becomes increasingly challenging at lower supply voltages. In literature, VCO-based ADCs are the most common timedomain circuits studied and developed for both low- and highfrequency applications [13], [14]. Additionally, in recent years time-based amplifiers [23] and neural network circuits [24] have also been proposed.

The conventional single-phase open-loop VCO ADC block diagram is shown in Fig. 2 [25]. A sensor connected to the ADC input generates a voltage $v_{i n}(t)$ which is used as a control signal for the $\mathrm{VCO}$. The VCO converts the voltage

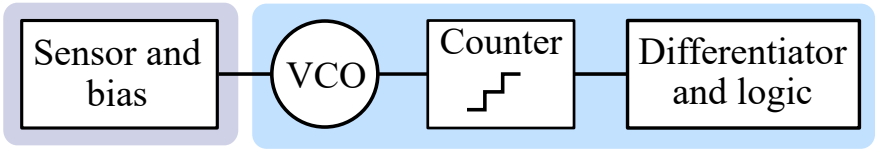

Fig. 2. Block diagram of an open-loop VCO-based ADC.

$v_{\text {in }}(t)$ to time/phase domain where the conversion relationship (to a first-order approximation) is given by [26]:

$$
f_{v c o}(t)=f_{c}+K_{v c o} v_{i n}(t)
$$

where $K_{V C O}$ is the VCO frequency gain and $f_{c}$ is the free running frequency, defined as the VCO operating frequency when $v_{i n}(t)=0 \mathrm{~V}$. A digital counter is used to count the number of rising edges within one sampling period at the VCO output, limiting the phase quantization step to $2 \pi$ for the single-phase architecture shown in Fig. 2. The counter is followed by a differentiator and digital logic, which translate the number of rising edges into a digital code corresponding to $v_{\text {in }}(t)$. A quantization error is introduced by the counter since its output is sampled asynchronously relative to the VCO output. However, this quantization error (residual phase) inherently becomes the inital phase of the next sampling period, thus the VCO acts like an integrator. The combination of this time-domain integration, and the delta operation performed by the differentiator resemble a first-order Delta-Sigma $(\Delta \Sigma)$ modulator with a noise transfer function (NTF) [25], [26]:

$$
\operatorname{NTF}(z)=1-z^{-1}
$$

and a signal transfer function (STF):

$$
\operatorname{STF}(z)=K_{v c o} 2 \pi
$$

in z-domain.

Additionally, the VCO's phase noise has the same NTF as the quantization noise [25], [26]. This is an important feature for the STNO-based magnetic field-to-digital converter since typical STNOs have shown phase noise in the range from $-55 \mathrm{dBc} / \mathrm{Hz}$ to $-72 \mathrm{dBc} / \mathrm{Hz}$ at $1 \mathrm{MHz}$ offset, limiting their applications [15]. However, the STNO in the proposed architecture can replace both the sensor and VCO in Fig. 2 and acts as a magnetic field controlled oscillator. This unique characteristic in combination with the inherent noise shaping of open-loop VCO-based ADCs inspired us to explore the proposed architecture.

\section{Implementation and simulation setup}

The proposed architecture, presented in Fig. 3 consists of a bias-T, low-noise amplifier (LNA), a limiting amplifier chain and a digital core. The challenging aspect of this architecture is the relatively low output power of the emerging STNO devices, requiring amplification to drive the digital counter. Additionally, MTJ STNO measurements have typically shown a tuning range over a few $\mathrm{MHz} / \mathrm{Oe}$ while macrospin simulations have gone as high as $17 \mathrm{MHz} / \mathrm{Oe}$ for an optimized geometry [6]. This wide tunability of the STNO operating frequency requires that the LNA and amplifier chain in Fig. 3 have a wide bandwidth. To meet these challenging requirements, a balun 


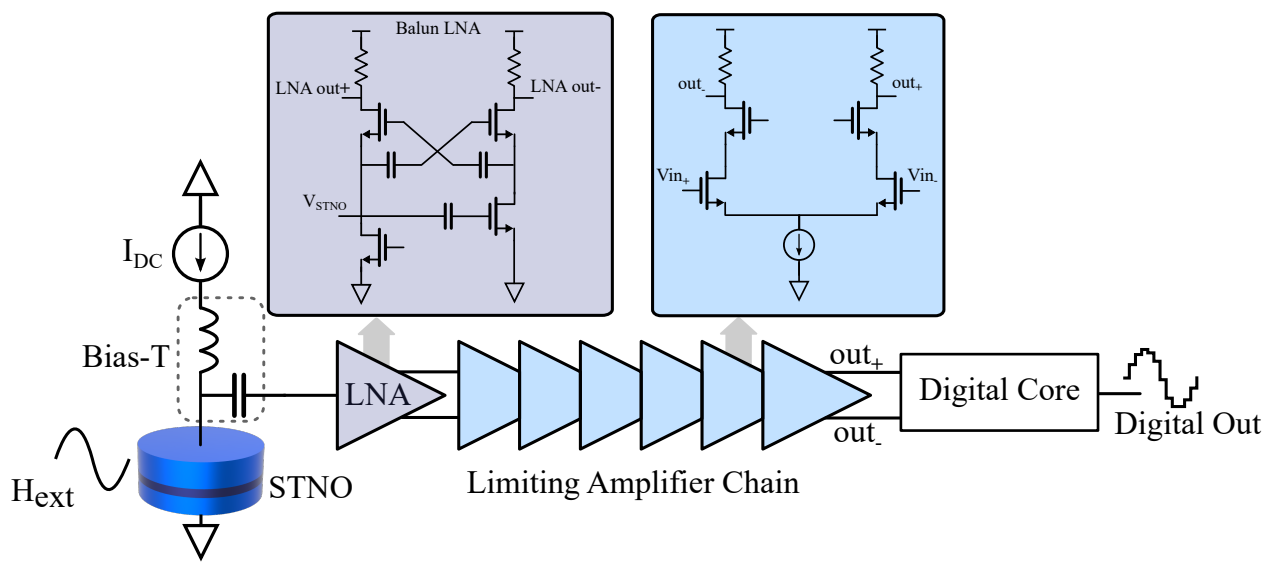

Fig. 3. The proposed STNO-based magnetic field-to-digital converter.

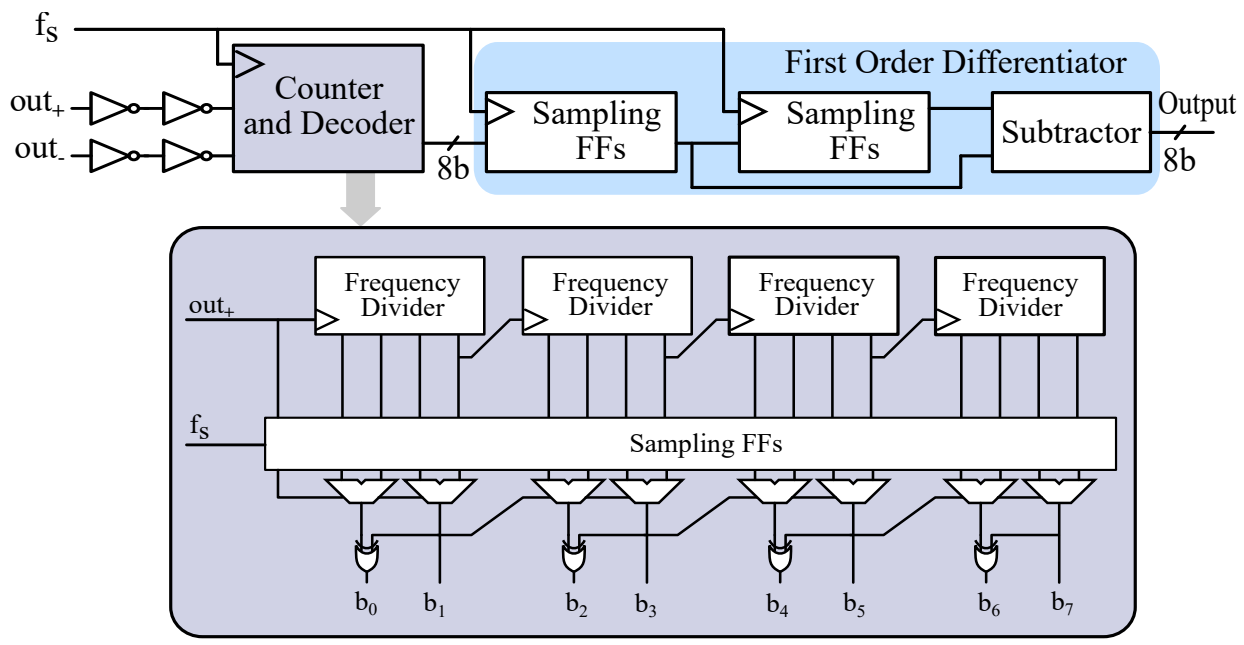

Fig. 4. Block diagram of the digital core and schematic of the asynchronous counter with double sampling and decoder.

LNA proposed in [27] and shown in Fig. 3 can be used. The balun LNA consists of a common-source (CS) stage and a cross coupled common-gate (CG) stage. This architecture provides an unbalanced to balanced conversion, gain boosting, bandwidth enhancement and good trade-offs in terms of gain balancing, noise performance and matching. The balun LNA is followed by a limiting amplifier chain consisting of five cascode stages to further enhance the gain, and an output CS stage allowing for a large output swing able to drive the digital core [27]. The digital core is presented in Fig. 4 were the first stage consists of a ripple counter and a decoder inspired by the architecture proposed in [14]. Since the ripple counter operates asynchronously relative to the sampling clock, $f_{s}$, this architecture is specifically designed to prevent metastability when sampling the counter value. This is achieved by doublesampling the outputs of the frequency divider and employing a series of MUXes to choose sampled values that are guaranteed to be stable. The chosen sampled values are then decoded using xor gates before being passed to the first-order differentiator, finally producing a digital representation of the magnetic field, $H_{e x t}$. It is worth mentioning that frequency dividers in
Fig. 4 are implemented with fully differential latches but are here depicted as single ended for simplicity.

Individual blocks in Fig. 3 have been tested and validated separately by using co-simulations. Specifically, the digital core has been design at the schematic level and tested together with the behavioral models of other blocks. However, attributed to the extensive simulation times, the system level performance (presented in Section III) is evaluated using Verilog-A/Verilog simulations of analog/digital blocks, without affecting the conclusion in terms of feasibility. The used MTJ STNO model is based on macrospin simulations [28]. The model is based on the MTJ STNO in [29] with a composition: $\operatorname{PtMn}(15) / \mathrm{Co}_{70} \mathrm{Fe}_{30}(2.5) / \mathrm{Ru}(0.85) /$ $\mathrm{Co}_{40} \mathrm{Fe}_{40} \mathrm{~B}_{20}(2.4) / \mathrm{MgO}(0.8) / \mathrm{Co}_{60} \mathrm{Fe}_{20} B_{20}(1.8)$ (thickness in $\mathrm{nm}$ ), which provides comprehensive information required for modeling. Additionally, this device has been selected due to its relatively high output power and reasonable phase noise. The STNO macrospin model takes into account critical characteristics, such as phase noise, and frequency and amplitude variation with $H_{e x t}$ and $I_{D C}$ [30], [21]. The MTJ STNO model parameters are given in Table I. A DC 
TABLE I

STNO AND LNA+AMPLIFICATION CHAIN SIMULATION PARAMETERS

\begin{tabular}{lll}
\hline Parameter & Definition & Value \\
\hline$H_{i n t}$ & Coupling field & $125 \mathrm{Oe}$ \\
$H_{A}$ & Anisotropy field & $120 \mathrm{Oe}$ \\
$H_{s}$ & Demagnetization field & $9500 \mathrm{Oe}$ \\
$\epsilon$ (Unitless) & Spin-polarization efficiency & 0.7 \\
q1 (Unitless) & First coefficient in $\alpha(\xi)$ expansion & 20 \\
$\eta$ (Unitless) & Noise power & 0.05 \\
$R_{a p}$ & Antiparallel resistance & $650 \Omega$ \\
$R_{p}$ & Parallel resistance & $300 \Omega$ \\
$\phi_{\text {ext }}$ & In-plane external field angle & $40^{\circ}$ \\
$\theta_{\text {ext }}$ & Out-of-plane external field angle & $0^{\circ}$ \\
$\alpha$ (Unitless) & Gilbert damping parameter & 0.018 \\
$\Delta_{t}$ & Time-step for phase noise generation & $10 \mathrm{ps}$ \\
$I_{D C}$ & Applied DC current & $1.5 \mathrm{~mA}$ \\
$W \times L$ & STNO area & $140 \mathrm{~nm} \times 85 \mathrm{~nm}$ \\
\hline$A_{v}$ & LNA+Amplifier chain gain & $\approx 35 \mathrm{~dB}$ \\
$\mathrm{BW}$ & LNA+Amplifier chain bandwidth & $3-8 \mathrm{GHz}$ \\
$\mathrm{NF}$ & Noise figure & $5 \mathrm{~dB}$ \\
$\mathrm{IIP} 3$ & Third order input intercept point & $-28 \mathrm{dBm}$ \\
\hline
\end{tabular}

current $I_{D C}=1.5 \mathrm{~mA}$ was chosen to maximize the output power while not exceeding the STNO breakdown voltage. The measurement results in [29] show a reasonable output power for in-plane angles in the range of $40^{\circ}<\phi_{\text {ext }}<80^{\circ}$ (enough to drive the digital core after amplification), while outside this region the power of the STNO fundamental rapidly decreases and the linewidth increases. Consequently, the proposed architecture will not function properly for in-plane field angles outside this range. Therefore, the in-plane and out-of-plane external field angles were chosen as $\phi_{e x t}=40^{\circ}$ and $\theta_{\text {ext }}=0^{\circ}$, respectively to achieve a high output power and low phase noise while the rest of the parameters where either extracted from [29] or used as fitting parameters. The MTJ STNO macrospin model does not take into account instantaneous changes in phase noise as a function of $H_{e x t}$ [21]. Therefore, in the following simulations the phase noise is assumed to be independent of $H_{e x t}$ with a linewidth $\Delta f=0.15 \mathrm{GHz}$. For a more detailed description of the STNO model and parameters in Table I, see [28], [30], [21]. For accurate modeling of the amplifying stages, important characteristics such as gain, bandwidth and noise figure are taken into account. The amplifier simulation parameters, presented in Table I, are inspired by the measurement results of the STNO amplifier in [27]. It is important to note that the bandwidth is between $3 \mathrm{GHz}$ and $8 \mathrm{GHz}$, which limits the STNO operating frequency to the same range. Lastly, as it was previously mentioned, Verilog descriptions were employed for the digital circuits. However, the functionality of the digital core was also verified up to 8 $\mathrm{GHz}$ in a $65 \mathrm{~nm}$ CMOS technology.

\section{RESULTS AND DISCUSSION}

The simulated STNO operating frequency, $f_{S T N O}$ as a function of the external magnetic field is presented in Fig. 5. The device exhibits a frequency shift of $5 \mathrm{GHz}$ over a change in external field of $700 \mathrm{Oe}$, resulting in a frequency tunability of $K_{S T N O} \approx 7 \mathrm{MHz} / \mathrm{Oe}$. The relationship is nearly linear for $H_{\text {ext }}>500 \mathrm{Oe}$, but for lower external fields it deviates significantly. This non-linearity significantly degrades the performance of the magnetic field-to-digital converter and

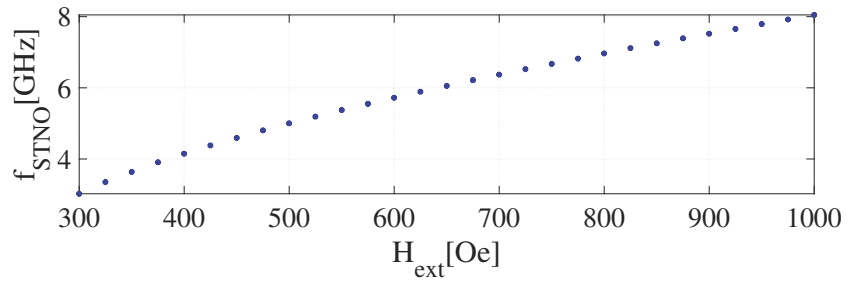

Fig. 5. STNO operating frequency as a function of external magnetic field.

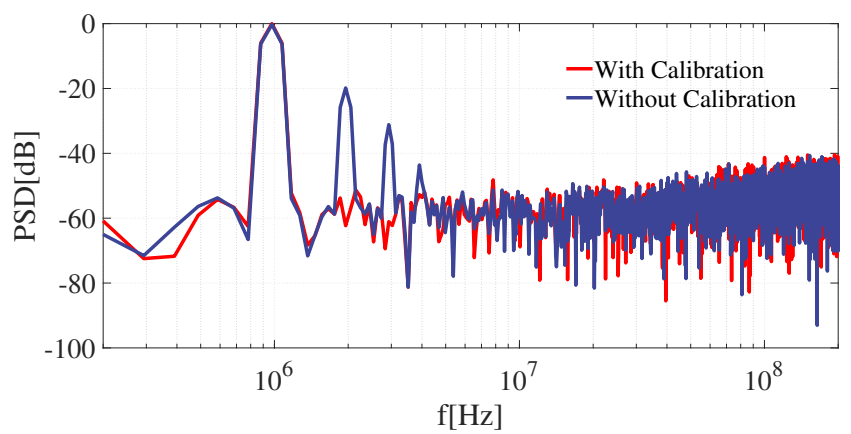

Fig. 6. Output spectrum of the VCO-based ADC before and after calibration for $f_{i n} \approx 1 \mathrm{MHz}, f_{s}=400 \mathrm{MHz}$ and NFFT $=4096$.

needs to be accounted for. To overcome this issue, a lookup-table (LUT) calibration method was introduced after the differentiator. This method is commonly used to account for non-linearities in open-loop VCO-based ADCs [14], [25]. The effect of the LUT calibration on the output spectrum of the STNO-based magnetic field-to-digital converter is shown in Fig. 6. Attributed to the extremely long simulation time of the STNO macrospin model, a low number of FFT points has been used. The performance of the proposed architecture is evaluated in terms of signal-to-noise and distortion ratio (SNDR), which directly translates to the effective number of bits (ENOB), similar to conventional ADCs. The SNDR as a function of the input bandwidth $f_{b w}$ for different oversampling ratios (OSR), OSR $=f_{s} / 2 f_{b w}$, where $f_{s}$ is the sampling frequency, is presented in Fig. 7. Here the external magnetic field, $H_{\text {ext }}$ is assumed to exhibit a sinusoidal shape with a peak value of $H_{e x t-p}=350 \mathrm{Oe}$ around a DC component of 650 Oe with a frequency of $f_{\text {in }} \approx 1 \mathrm{MHz}$. The SNDR is relatively independent of OSR for low $f_{b w}$, suggesting that phase noise dominates the achievable SNDR rather than quantization noise. To verify this assumption, we utilize the analytical equations which describe the VCO-based ADCs [25], [26], where the SNDR takes into account both quantization noise and STNO's phase noise:

$$
S N D R=10 \log _{10}\left(\frac{P_{s}}{P_{\text {dist }}+P_{n, \text { quant }}+P_{n, \text { STNO }}}\right)
$$

where $P_{\text {dist }}$ is the distortion, $P_{s}$ is the signal power defined as:

$$
P_{s}=0.5\left(\frac{H_{e x t-p} K_{S T N O} 2 \pi}{f_{s}}\right)^{2}
$$




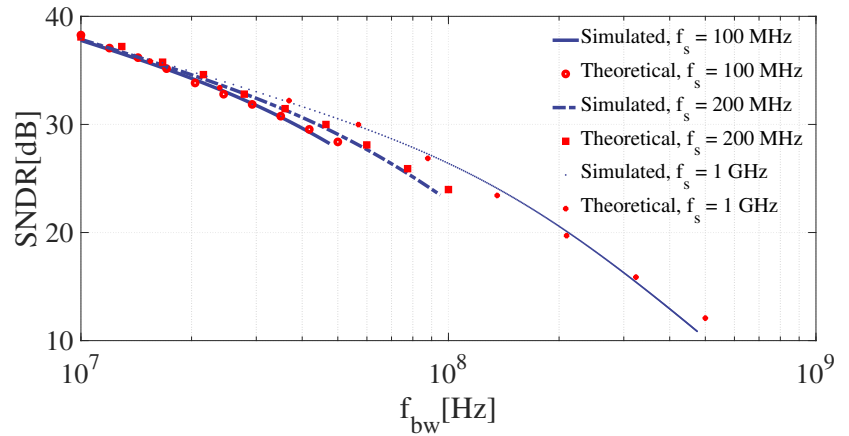

Fig. 7. Simulated and theoretical SNDR as a function of $f_{b w}$ for different $f_{s}, f_{\text {in }} \approx 1 \mathrm{MHz}$ and $H_{\text {ext }-p}=350 \mathrm{Oe}$.

$P_{n, q u a n t}$ is the quantization noise defined as:

$$
P_{n, q u a n t}=\frac{\pi^{4}}{9}\left(\frac{2 f_{b w}}{f_{s}}\right)^{3}
$$

and $P_{n, S T N O}$ is the STNO's phase noise:

$$
P_{n, S T N O}=\frac{32 \pi^{2} L \cdot f_{o f f}^{2} f_{b w}}{f_{s}^{2}}
$$

where $L$ is the phase noise at a frequency offset $f_{\text {off }}$.

In Eq. (9), $L=-53 \mathrm{dBc} / \mathrm{Hz}$ and $f_{\text {off }}=1 \mathrm{MHz}$ have been chosen for fitting the theoretical SNDR to the simulated SNDR. After introducing the LUT calibration technique, $P_{d i s t} \approx 0$ in Eq. (6), as it can be seen in Fig. 6 (the spurs are suppressed into the noise floor). The fitting results presented in Fig. 7 demonstrate that the theoretical SNDR match well the simulated SNDR. Additionally, the theoretical expressions allowed us to observe that for low $f_{b w}, P_{n, S T N O}>>P_{n, q u a n t}$, which confirms that the achievable SNDR is limited by phase noise. Consequently, increasing the OSR has negligible effect in the low $f_{b w}$ region. However, as the bandwidth increases, $P_{n, q u a n t}$ also increases as it can be seen from Eq. (8), making SNDR dependent also on quantization noise and OSR. The SNDR as a function of the input power for $f_{s}=200 \mathrm{MHz}$ is presented in Fig. 8 showing a dynamic range of $D R \approx 37 \mathrm{~dB}$. This dynamic range is limited by the bandwidth of the LNA and amplifier chain (3-8 GHz) as it was previously mentioned. Finally, the simulated SNDR as a function of $f_{i n}$ for a fullscale input showed a relatively constant SNDR with $f_{i n}$ in the range $0.5-5 \mathrm{MHz}$, consistent with the theory in Eq. (6)-(9). As it can be seen in Fig. 8, the maximum achievable SNDR is $\approx 38 \mathrm{~dB}$, which translates to:

$$
E N O B=\frac{S N D R-1.76}{6.02} \approx 6
$$

for the proposed architecture. These simulation results validate the feasibility of the proposed magnetic field-to-digital converter and indicate that its performance is limited by the STNO phase noise characteristics.

The performance can be enhanced considering the current development in the STNO technology. For instance, the magnetic field's dynamic range could be tailored to a specific application utilizing other materials and/or by changing the STNO's size. Alternatively, lower phase noise and higher output power

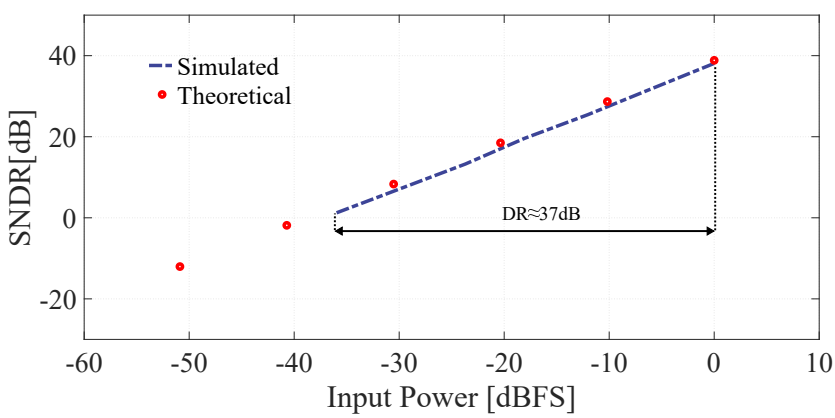

Fig. 8. Simulated SNDR and theoretical SNDR as a function of input power for $f_{i n} \approx 1 \mathrm{MHz}, f_{s}=200 \mathrm{MHz}$ and $f_{b w}=10 \mathrm{MHz}$.

MTJ STNO's have been demonstrated by utilizing vortex oscillations in STNOs [15]. Moreover, vortex based STNOs usually exhibit lower operating frequencies (less than $2 \mathrm{GHz}$ ) [15], relaxing the design constraints of the amplifier chain and digital logic. However, they currently show a lower tunability compared to other spintronic oscillators. In recent years, arrays of synchronized spintronic oscillators, which have shown a reduction in phase noise and improvements in output power, have also gained attention [31].

\section{CONCLusion}

In this work, a magnetic field-to-digital converter based on STNOs was proposed. Schematic simulations of individual blocks and behavioral simulations of the whole architecture were carried out, demonstrating its feasibility. The achievable SNDR is fundamentally limited by the STNO phase noise, similar to conventional VCO-based ADCs. However, with the extensive research focused on increasing the output power and reducing the phase noise of spintronic oscillators, the magnetic field-to-digital converter performance could be significantly improved in future implementations.

\section{REFERENCES}

[1] C. Tsang et al., "Design, fabrication and testing of spin-valve read heads for high density recording," IEEE Transactions on Magnetics, vol. 30, no. 6, pp. 3801-3806, Nov 1994.

[2] C. C. Huang, X. Zhou, and D. A. Hall, "Giant Magnetoresistive Biosensors for Time-Domain Magnetorelaxometry: A Theoretical Investigation and Progress Toward an Immunoassay,' Scientific Reports, vol. 7, no. April, pp. 1-10, 2017.

[3] T. Hirata et al., "Development of a highly sensitive magnetic field detector with a wide frequency range for nondestructive testing using an hts coil with magnetic sensors," IEEE Transactions on Applied Superconductivity, vol. 29, no. 5, pp. 1-5, Aug 2019.

[4] J. Lenz and A. S. Edelstein, "Magnetic sensors and their applications," IEEE Sensors Journal, vol. 6, no. 3, pp. 631-649, 2006.

[5] P. Ripka and M. Janosek, "Advances in Magnetic Field Sensors," Journal of Physics: Conference Series, vol. 10, no. 6, pp. 1108-1116, 2010.

[6] P. M. Braganca, B. A. Gurney, B. A. Wilson, J. A. Katine, S. Maat, and J. R. Childress, "Nanoscale magnetic field detection using a spin torque oscillator," Nanotechnology, vol. 21, no. 23, 2010.

[7] H. S. Lee et al., "Power-efficient spin-torque nano-oscillator-based wireless communication with cmos high-gain low-noise transmitter and receiver," IEEE Transactions on Magnetics, vol. 55, no. 5, pp. 1-10, May 2019.

[8] M. Romera et al., "Vowel recognition with four coupled spin-torque nano-oscillators," Nature, vol. 563, no. 7730, pp. 230-234, 2018. 
[9] D. Fan, S. Maji, K. Yogendra, M. Sharad, and K. Roy, "Injection-locked spin hall-induced coupled-oscillators for energy efficient associative computing," IEEE Transactions on Nanotechnology, vol. 14, no. 6, pp. 1083-1093, Nov 2015.

[10] J. G. Zhu, X. Zhu, and Y. Tang, "Microwave assisted magnetic recording," IEEE Transactions on Magnetics, vol. 44, no. 1, pp. 125-131, 2008.

[11] J.-g. Zhu and Y. Wang, "Microwave Assisted Magnetic Recording Utilizing Perpendicular Spin Torque Oscillator With Switchable Perpendicular Electrodes," IEEE Transactions on Magnetics, vol. 46, no. 3, pp. 751757,2010

[12] T. Chen, S. Rodriguez, J. Åkerman, and A. Rusu, "An inductorless wideband balun-lna for spin torque oscillator-based field sensing," in 2014 21st IEEE International Conference on Electronics, Circuits and Systems (ICECS), Dec 2014, pp. 36-39.

[13] M. Z. Straayer and M. H. Perrott, "A 12-bit, 10-MHz bandwidth, continuous-time $\Sigma \Delta$ ADC with a 5-bit, 950-MS/s VCO-based quantizer," IEEE Journal of Solid-State Circuits, vol. 43, no. 4, pp. 805-814, 2008.

[14] M. Baert and W. Dehaene, "20.1 a 5gs/s 7.2 enob time-interleaved vcobased adc achieving 30.5fj/conv-step," in 2019 IEEE International SolidState Circuits Conference - (ISSCC), Feb 2019, pp. 328-330.

[15] T. Chen et al., "Spin-torque and spin-hall nano-oscillators," Proceedings of the IEEE, vol. 104, no. 10, pp. 1919-1945, Oct 2016.

[16] J. Slonczewski, "Current-driven excitation of magnetic multilayers," Journal of Magnetism and Magnetic Materials, vol. 159, no. 1-2, pp. L1-L7, 1996.

[17] J. C. Slonczewski, "Excitation of spin waves by an electric current," Journal of Magnetism and Magnetic Materials, vol. 195, no. 2, pp. 261-268, 1999.

[18] E. B. Myers, D. C. Ralph, J. A. Katine, R. N. Louie, and R. A. Buhrman, "Current-induced switching of domains in magnetic multilayer devices," Science, vol. 285, no. 5429, pp. 867-870, 1999.

[19] M. Julliere, "Tunneling between ferromagnetic films," Physics Letters A, vol. 54, no. 3, pp. 225-226, 1975.

[20] J. G. J. Zhu and C. Park, "Magnetic tunnel junctions," Materials Today, vol. 9, no. 11, pp. 36-45, 2006.

[21] T. Chen et al., "Comprehensive and macrospin-based magnetic tunnel junction spin torque oscillator model- part ii: Verilog-a model implementation," IEEE Transactions on Electron Devices, vol. 62, no. 3, pp. 1045-1051, March 2015.

[22] P. K. Muduli, O. G. Heinonen, and J. Åkerman, "Bias dependence of perpendicular spin torque and of free- and fixed-layer eigenmodes in MgO-based nanopillars," Physical Review B - Condensed Matter and Materials Physics, vol. 83, no. 18, pp. 28-30, 2011.

[23] K. Kim and C. Yoo, "Time-domain operational amplifier with voltagecontrolled oscillator and its application to active-rc analog filter," IEEE Transactions on Circuits and Systems II: Express Briefs, pp. 1-1, 2019.

[24] D. Miyashita, S. Kousai, T. Suzuki, and J. Deguchi, "Time-domain neural network: A 48.5 tsop/s/w neuromorphic chip optimized for deep learning and cmos technology," in 2016 IEEE Asian Solid-State Circuits Conference (A-SSCC), Nov 2016, pp. 25-28.

[25] J. Kim, T. Jang, Y. Yoon, and S. Cho, "Analysis and design of voltage-controlled oscillator based analog-to-digital converter," IEEE Transactions on Circuits and Systems I: Regular Papers, vol. 57, no. 1, pp. 18-30, Jan 2010.

[26] E. Sacco, J. Vergauwen, and G. Gielen, "From open-loop to closed-loop single-vco-based sensor-to-digital converter architectures: theoretical analysis and comparison," in 2019 IEEE 8th International Workshop on Advances in Sensors and Interfaces (IWASI), June 2019, pp. 29-34.

[27] T. Chen, P. Dürrenfeld, S. Rodriguez, J. Åkerman, and A. Rusu, "A highly tunable microwave oscillator based on mtj sto technology," Microwave and Optical Technology Letters, vol. 56, no. 9, pp. 20922095, 2014.

[28] A. Slavin and V. Tiberkevich, "Nonlinear auto-oscillator theory of microwave generation by spin-polarized current," IEEE Transactions on Magnetics, vol. 45, no. 4, pp. 1875-1918, April 2009.

[29] Z. M. Zeng et al., "Enhancement of microwave emission in magnetic tunnel junction oscillators through in-plane field orientation," Applied Physics Letters, vol. 99, no. 3, pp. 1-4, 2011.

[30] T. Chen et al., "Comprehensive and macrospin-based magnetic tunnel junction spin torque oscillator model-part i: Analytical model of the mtj sto," IEEE Transactions on Electron Devices, vol. 62, no. 3, pp. 1037-1044, March 2015.

[31] M. Zahedinejad et al., "Two-dimensional mutually synchronized spin Hall nano-oscillator arrays for neuromorphic computing," Nature Nanotechnology, 2019.

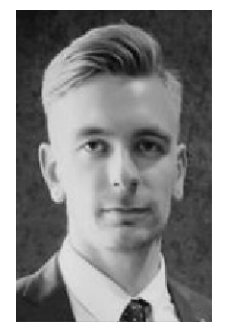

Dagur Ingi Albertsson received the B.Sc. degree in mechatronics engineering from the University of Reykjavik, Reykjavik, Iceland, in 2016, and the M.Sc. degree in embedded systems from KTH Royal Institute of Technology, Kista, Sweden, in 2018, where he is currently pursuing the Ph.D. degree, with the research area of circuit design for spin torque and spin hall nano oscillators.

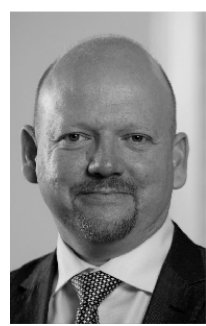

Johan Åkerman received the Ing. Phys. Dipl. degree from EPFL, Zurich, Switzerland, in 1994, the M.Sc. degree in physics from Lund University, Lund, Sweden, in 1996, and the Ph.D. degree in materials physics from KTH Royal Institute of Technology, Stockholm, Sweden, in 2000. In 2008, he was appointed Full Professor at the University of Gothenburg, Gothenburg, Sweden and is a Guest Professor at the KTH Royal Institute of Technology. $\mathrm{He}$ is also the founder of NanOsc $\mathrm{AB}$ and NanOsc Instruments $\mathrm{AB}$, Kista, Sweden.

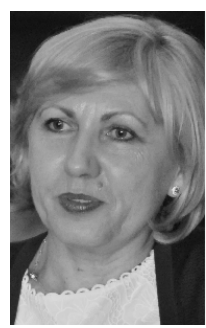

Ana Rusu received the M.Sc. degree in electronics and telecommunications from the Technical University of Iasi and the Ph.D. degree in electronics engineering from the Technical University of ClujNapoca, Romania, in 1983 and 1998, respectively. Since September 2001, she has been with the KTH Royal Institute of Technology Stockholm, Sweden, where she is a professor in integrated circuits and systems. Her current research interests span from low/ultra-low power high performance CMOS circuits and systems for biomedical applications to emerging technologies, such as STO-based systems and monolithic 3D integration technology, and high temperature SiC BJT circuits. 